FORUM FORUM
FORUM

FORUM is intended for new ideas or new ways of interpreting existing information. It provides a chance for suggesting hypotheses and for challenging current thinking on ecological issues. A lighter prose, designed to attract readers, will be permitted. Formal research reports, albeit short, will not be accepted, and all contributions should be concise with a relatively short list of references. A summary is not required.

\title{
Trend words in ecological core journals over the last 25 years (1978-2002)
}

\author{
Michael Nobis and Thomas Wohlgemuth, Swiss Federal Research Institute WSL, Zürcherstr. 111, CH-8903 \\ Birmensdorf, Switzerland (michael.nobis@wsl.ch).
}

Ecological research has been repeatedly criticized for its general lack of progress. While it has proved difficult to reveal scientific progress, from a subjective view trends in ecology seems evident. In order to objectively detect principal trends in this broad discipline during the past 25 years (1978-2002), we performed several word and co-word analyses on titles and abstracts of articles that appeared in five core journals of ecology: «Ecology», «Oecologia», «Oikos», «Journal of Animal Ecology» and «Journal of Ecology». A general time gradient in the use of words was evident and words with a positive trend dominated. Trend words derived from article titles were used to perform coword analyses on abstracts available since 1991. Besides a trend towards the study of processes most important ecological fields found are landscape ecology, biodiversity, ecosystem research, modelling, and food-web/predator-prey interactions. A semantic map of trend words was built by ordination. In the map, words formed a continuous space with the three thematic directions «biotic», «abiotic» and «temporal \& spatial» representing the classic aspects of ecological research. With respect to the obvious trends and features derived from our analyses, we suggest that the results represent real scientific progress in ecology as a pluralistic, multi-aspect and multiple-causation discipline. Such a progress could hardly be observed from details in sub-disciplines.

Over the last years, there has been a lively debate on how ecology has progressed (Lawton 1991, 1999, Peters 1991, Grime 1993, Ghilarov 2001, Oksanen et al. 2001, Swihart et al. 2002). Part of the discussion is the difficulty of how to measure real scientific progress (Weiner 1995, 1999, Aarssen 1999). We approach such a measure by asking whether there are trends expressed by the choice of words in ecology as the pluralistic multiplecausation discipline (Paine 2002). Clearly, assessing scientific trends is a key-activity among scientists. Evidence for this are the high rates of trend-setting journals such as «Nature», «Science» or «Trends in Ecology and Evolution». Different reasons have been accounted for scientific trends in ecology: The introduction of new technologies and methodological innovations (Oksanen 1991, Wiens 1992), the adoption of new principles, theories or paradigms (Kuhn 1962, Naeem 2002), the use of fads or buzz-words such as sustain- ability and biodiversity to getting projects funded (Ghilarov 1996, Shurin et al. 2001). In addition, obvious environmental anomalies can force trends in ecological research (Odén 1968, Keeling et al. 1976). However, only by retrospective analysis of a series of indicators the importance of once important-looking trends can be carefully evaluated and eventually be assessed for progress.

Topicality and scientific trends have been sensed in many ways: by counting the number of ecological papers per year or the number of references per paper (Graham and Dayton 2002), by questioning scientists on key ideas of ecology and analysing the responses (Cherrett 1989), by inviting renowned experts to write on trends of their disciplines (Ambasht 1998, Nagy et al. 2003), by publishing proceedings of meetings aimed at assessing trends of a discipline (Maruyama et al. 1991, Körner and Spehn 2002, Manel et al. 2003), by joining several authors in a special journal feature (Graham and Dayton 2002), by comparing textbooks on ecological disciplines (Woodward 1987, Paine 2002), by reviewing new textbooks in journals or by publishing papers in journals dedicated to reporting the most exciting current research. Additional effective assays can be found in scientific grant proposals and in papers submitted for publication. However, these indicators are often confidential and thus not available for analysis by the community. Other indicators include topics of conferences, congresses and workshops. A consolidated trend is often expressed in the foundation of new journals (Graham and Dayton 2002) - sometimes as a spin-off of traditional journals, e.g. «Global Ecology and Biogeography» derived from «Journal of Biogeography». Trend subjects in scientific papers are usually observable by the mutated combination of words, by new compound words or even by a new terminology. Word analyses of publications were applied to detect longterm trends in animal ecology (Shorrocks 1993), to sense 
the actual use of mathematical tools in ecology (Budilova et al. 1997) and to study scientific progress in the frame of one ecological journal (Swihart et al. 2002).

In this study, we conducted a word analysis of titles and abstracts of five ecological core journals spanning a 25-year period. In order to assess current and past trends in the wide field of ecology, we focused on a traditional body of ecological journals that a) have ecology as the main focus, b) publish a large annual number of papers, and c) that have been highly rated by the Institute for Scientific Information (ISI, Philadelphia, PA, USA). We addressed the following questions:

- Does a word analysis reveal a general trend in the use of title words in ecological core journals over the last 25 years $(1978-2002)$ ?

- Which words have shown the strongest positive trends in usage?

- Which specific trends in ecology are represented by the trend words found?

\section{Methods}

\section{Material}

The trend analysis is based on five ecological core journals and covers a 25-year period (1978-2002). Three out of these five journals provide general ecological orientation: «Ecology», «Oecologia», «Oikos». In two additional journals, ecology is focussed on either zoological or botanical fields: «Journal of Animal Ecology» and «Journal of Ecology». Journals that are specialized in specific fields related to ecology such as «Landscape Ecology», «Journal of Applied Ecology» or «Ecological Modelling» were not considered here.

We used the ISI web of Science to obtain titles of all papers that appeared in the issues of the years 1978-2002 of the five journals. Abstracts were consistently available since 1991. For publications classified in the ISI web of Science as «articles» the titles and abstracts were parsed into single strings and stored in a database (19.010 publications; 238.976 title strings; 2.515.856 abstract strings). For convenience, we applied «word» for any string mentioned below.

\section{Word processing}

Titles and abstracts were split into words. Characters

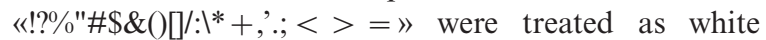
space and separators. The problem of compound words was treated as follows: a) we searched for compound words made up of two words and separated by a hyphen, such as fresh-water, b) we searched for same consecutive words «fresh water» and replaced the space by a hyphen, c) we searched for same compound words «freshwater» and split the word into «fresh-water». Plurals of words were treated as singulars. In consequence, words were reduced by the ending «s» if the resulting words already existed in the database, i.e. that also differently conjugated verbs were joined. The same procedure was applied for words ending in «y» and «ies». The database was restricted to words with at least three letters or digits. Because of obvious changes in the use of the

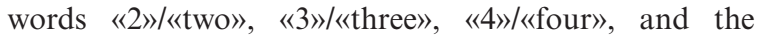
inconsistent use of the words «comment» and «reply», these words were excluded from the data-sets. American and British spellings were unified. Syllable divisions in abstracts, e.g. «avail-ability»/〈availability», were ignored. The final database consisted of 179.152 title words and 1.919.080 abstract words.

\section{Data-sets and data processing}

We created four data-sets in order to focus step-by-step on possible trends. A first data-set (A) was defined for the simultaneous analysis of the differences between the journals and publication years. It is based on most frequent title-words (i.e. words appearing 25 times and more between 1978 and 2002). Counts of words refer to numbers per journal and year. Because these numbers and the title lengths fluctuated and generally increased for all journals, we defined

$\mathrm{n}_{\mathrm{ijy}}^{\prime}=1000 \times \mathrm{n}_{\mathrm{ijy}} / \mathrm{N}_{\mathrm{jy}}$

where $n_{i j y}$ is the number of a word $i$ in the titles of a certain journal and year. Here $\mathrm{N}_{\mathrm{jy}}$ is the total number of all title-words for this journal and year. $\mathrm{n}_{\mathrm{ijy}}^{\prime}$ was used in the subsequent analysis. Data-set A consists of the 1.064 most frequent title-words corresponding to 127.475 occurrences in 125 journal-year observations.

In order to focus on general trends over time, a second data-set (B) merely contains the annual counts of titlewords in data-set A without distinction by journals, resulting in 1.064 most frequent title-words in 25 year observations. As with data-set $\mathrm{A}$, the counts were divided by the total annual number of words.

In order to find semantic connections between single trend words from previous results we built two additional data-sets $\mathrm{C}$ and $\mathrm{D}$ that served for co-word analyses. Using the most important words having a positive trend in publication titles, the two matrices were defined by presence-absence co-occurrences of these words within the available abstracts from 1991 to 2002 (9.814 abstracts, 1.919.080 words).

Data-set C (co-word analysis 1) was calculated as symmetric similarity matrix using the Yule-index (Podani 1994) for a small list of most important title words with a positive trend (results section): 


$$
\mathrm{YUL}=(\mathrm{ad}-\mathrm{bc}) /(\mathrm{ad}+\mathrm{bc})
$$

where $\mathrm{a}$ is the number of abstracts where both words occur, $b$ and $c$ the abstracts having only one of the two words and $\mathrm{d}$ the number of abstracts without the two.

Data-set D (co-word analysis 2) was defined as asymmetric matrix, by using a list of 150 title words with a strong positive trend as columns, and another enlarged list with about the double number of title words with a positive trend as rows (results section). Numbers of abstracts for the occurrences of every word combination were counted and divided by the abstract counts for the corresponding words belonging to the larger second list. In both data-sets $\mathrm{C}$ and $\mathrm{D}$, zero-values were used for co-occurrences of identical words in columns and rows.

The trend analysis and the search for semantic word groups was performed by multivariate ordination and classification. We used principle components analysis (PCA) and correspondence analysis (CA) provided by the CANOCO 4.5 software (ter Braak and Smilauer 2002). Ordinations were performed after log-transformations. Word scores in PCA were post-divided by the standard deviation of each word in the data-sets. Most important words to display in scatter plots were selected by their fit to the underlying ordination-model. In PCA this fit corresponds with the linear regression coefficient for the word counts and the scores of the axes used as predictor variables. In CA the fit is based on a weighted regression (ter Braak and Smilauer 2002). For the coword analysis we additionally used a modified single linkage classification. The modification includes the iterative recalculation of the resemblance matrix after every fusion of two clusters. It reduces the chaining effect that is well known in classic single linkage clustering (Podani 1994) and improves the results in comparison to average or complete clustering.

\section{Results}

Between the years 1978 and 2002, a general increase occurred in the number of articles ( $\mathrm{p} \ll 0.001$ for linear model) and the number of words per article title ( $\mathrm{p} \ll 0.001$ for linear increase of non-averaged title lengths; Fig. 1). In addition, between the years 1991 and 2002, the length of abstracts increased ( $p \ll 0.001$ for linear increase of non-averaged abstract lengths).

\section{Trend words}

Differences in title word frequencies among journals and publication years are displayed as ordination bi-plot in Fig. 2. The first PCA axis on data-set A differentiates between zoological and botanical contents of article titles, principally reflecting the different emphases of the zoological «Journal of Animal Ecology» and the bota-
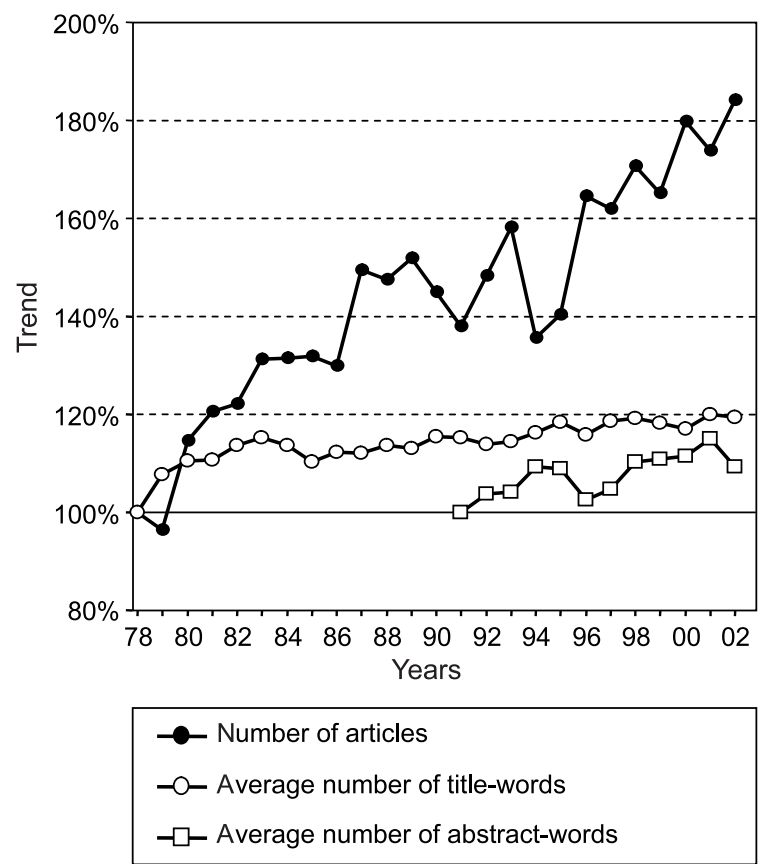

Fig. 1. Trend of articles per year $\left(\mathrm{n}_{1978}=52\right)$, average number of words per article $\left(\mathrm{n}_{1978}=8,2\right)$ and number of words per abstract $\left(\mathrm{n}_{1978}=175\right)$. Data refer to articles that appeared between 1978 and 2002 in the journals «Ecology», «Oecologia», «Oikos», «Journal of Animal Ecology» and «Journal of Ecology».

nical «Journal of Ecology» (Fig. 2A). The three journals «Oikos», «Ecology» and «Oecologia» are located at the centre of the first axis that corresponds to the general orientation expressed by the journal's title. However, «Oikos» shows a modest tendency towards zoological words ( $\mathrm{p}<0.05$; mean of first axis scores tested by 10.000 random permutations of journal assignment). Different grayscales for the succeeding five-year periods (Fig. 2B) reveal the second axis as an indirect time-axis. For each journal, a clear time gradient is obvious from the first time-period (1978-1982; top) to last time-period (1998-2002; bottom).

The PCA-ordination on data-set $\mathrm{B}$ resulted in the biplot in Fig. 3. Here, the words mentioned in the five journals were pooled for each year. As a result the indirect time gradient is now dominating the first two axes, which is expressed by a horseshoe-effect. The 150 most important words, i.e. words with best fits, are listed with respect to their positions in quadrants I, II, III and IV. Words with a positive trend are located in quadrant IV (297 in total, 66 displayed), those with a negative trend are found in quadrant I.

\section{Combination of trend words}

Co-word analysis 1 was carried out for the 66 words displayed in quadrant IV of Fig. 3. Based on the co- 


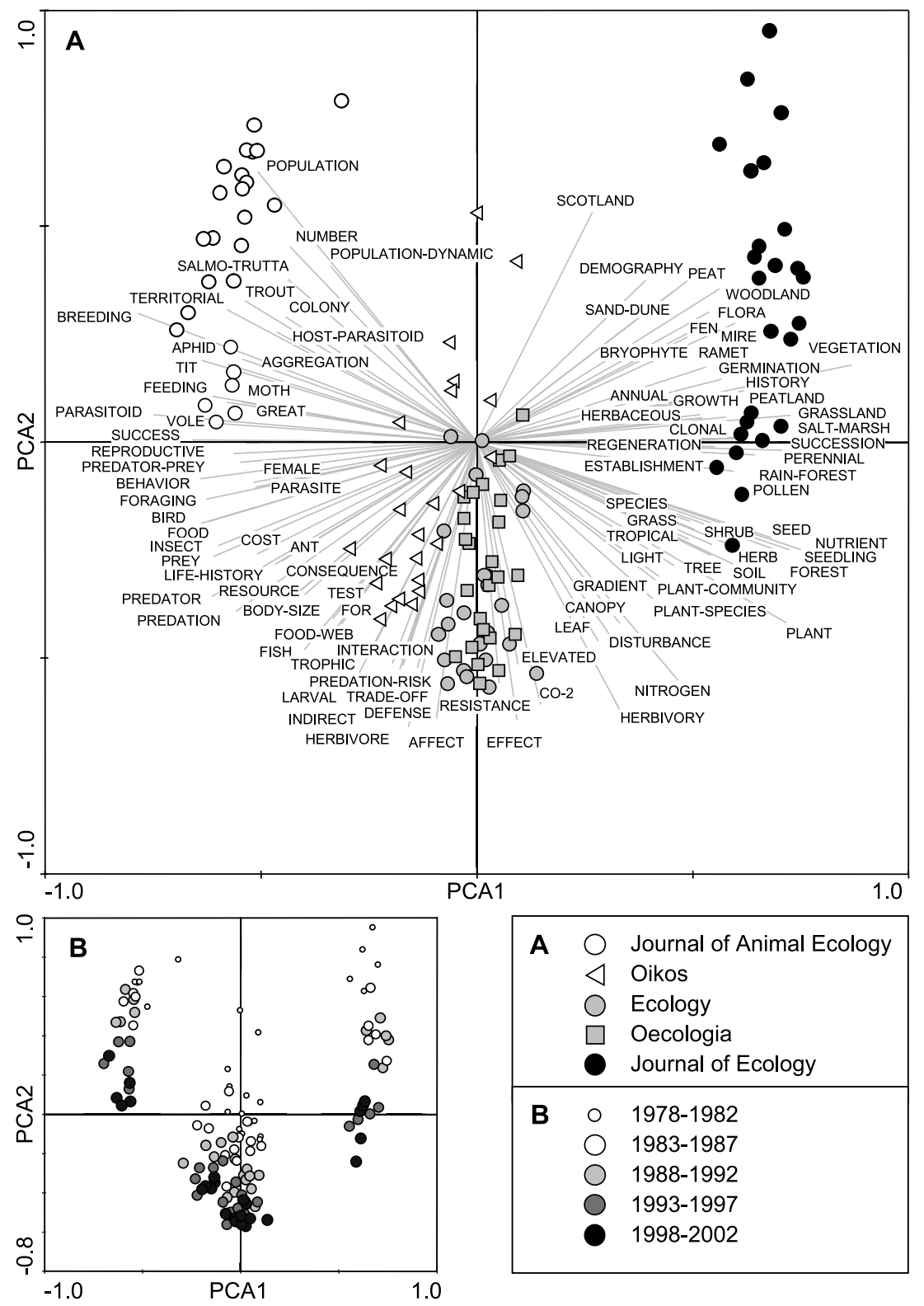

Fig. 2. PCA-biplot of data-set A using log-transformed, relative counts of title words per journal and year. Transformation: $\mathrm{n}^{\prime}=\log (\mathrm{n}+1)$; the first and the second PCA-axes accumulate $9.9 \%$ and $5.6 \%$ of total variance, respectively.

occurrences in abstracts between 1991 and 2002 (data-set C), several clusters proved interpretable as semantic coherent groups (Fig. 4). In order to compare the importance of the words in publication titles at the beginning and at the end of the 25 year period, relative frequencies are given with respect to the first and last five years, i.e. 1978-1982 and 1998-2002. For the coword analysis 2, the 150 best fitted title words in quadrant IV of Fig. 3 were compared with all title words of the same quadrant $(n=297)$. Figure 5 shows the scatter plot of the correspondence analysis of this cooccurrence-matrix (data-set D). Only the 100 best fitting words (out of 150) are displayed. Words in the scatter plot build a triangular data structure with the three main orientations towards the corners: «abiotic», «biotic» and «temporal and spatial». In the Appendix, five-year frequencies of the 25 best fitted title words of quadrant IV in Fig. 3 are listed with counts for the five journals.

\section{Discussion}

The results reveal a predominant and distinct time gradient in the use of title words in ecological publica- 
Fig. 3. PCA-biplot of of data-set $\mathrm{B}$ using log-transformed, relative counts of title words per year.

Transformation: $\mathrm{n}^{\prime}=\log (\mathrm{n}+1)$; the first and the second PCA-axes accumulate $23.7 \%$ and $7.6 \%$ of total variance, respectively. Best fitted 150 title words are displayed, top 50 words are in bold.
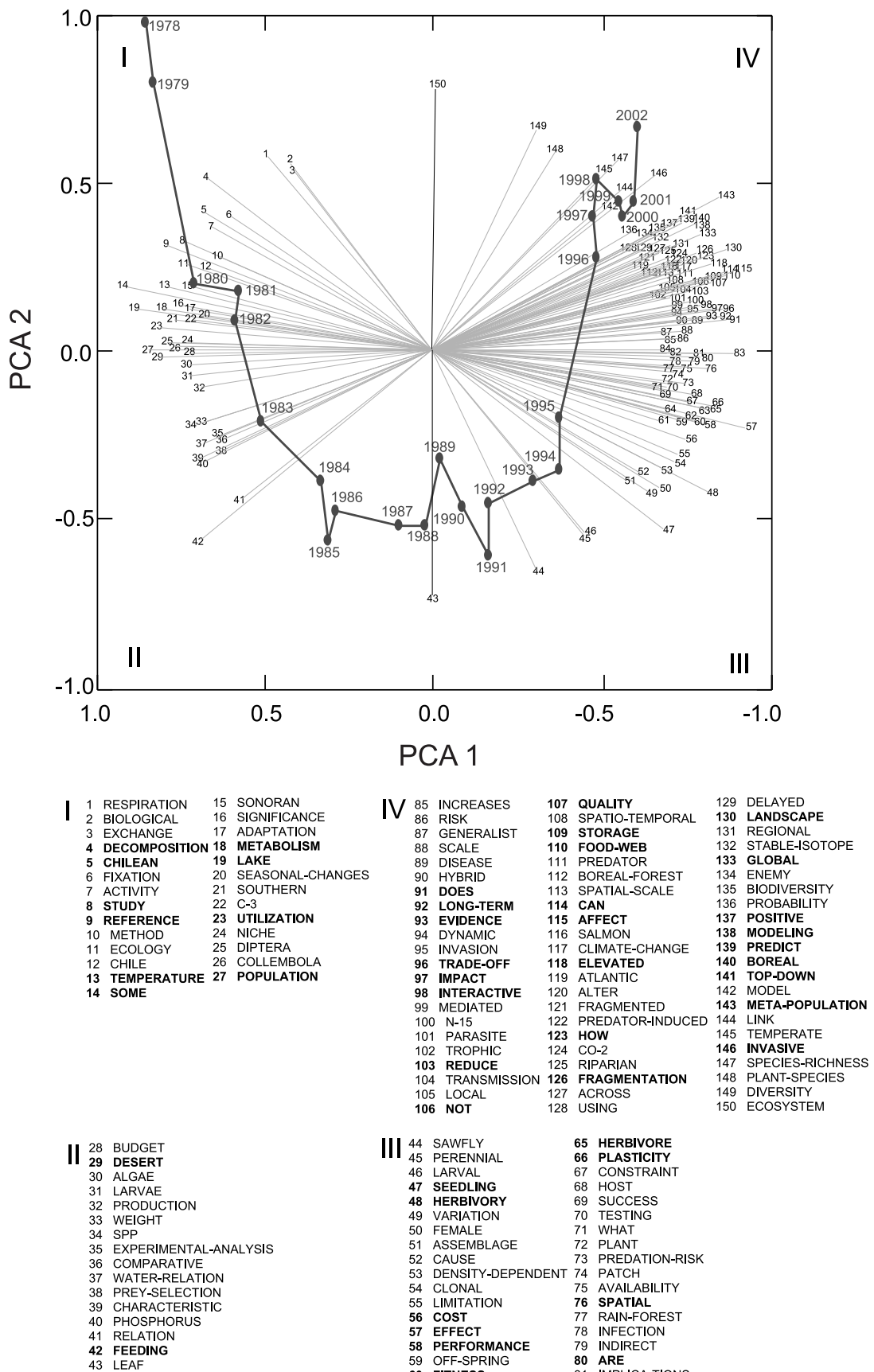

tions considered. Because this trend is consistent in each of the five journals we assume a general validity. The word analysis of the ecological core journals revealed an ongoing development of the use of words in research that is expressed by quasi-continuous steps along a general direction. These findings contradict the stagnation of ecological research (O'Connor 2000) and correspond to those comparable studies of Shorrocks (1993) and Swihart et al. (2002). At first sight, it seems plausible that a mutated use of ecological expressions cause a 


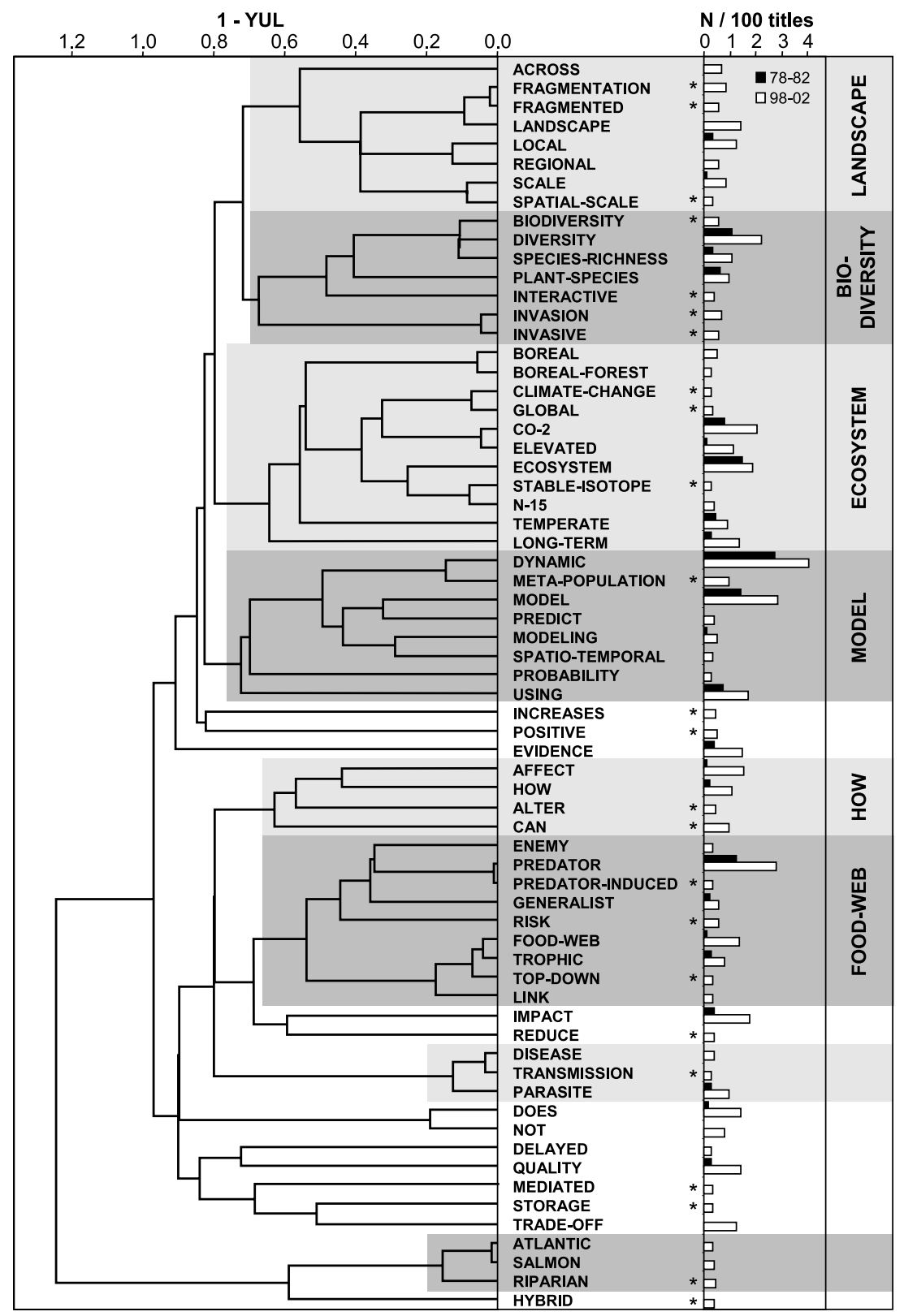

Fig. 4. Dendrogram of the 66 best fitted title words according to quadrant IV in Fig. 3, resulting from co-word analysis with Yuleindex (Podani 1994) and modified single-linkage clustering. Bars at the right indicate relative word frequencies in five ecological core journals with respect to the first and last five years, i.e. $1978-1982$ and 1998-2002. Absences with respect to the first period are indicated by stars.

prominent time gradient - even without scientific progress. However, an examination of all words shows that such a time gradient is also visible in words with no self-evident ecological meaning. Titles including «some» decreased, whereas questions containing «does», «can» and in particular «how» increased over time. Beside possible changes in the style, this may indicate a decline of descriptive titles, which, in return, reflects a decline in exclusive descriptive research (Shorrocks 1993). In contrast, titles concerning mechanisms and explanations have become more frequent indicating a trend towards the examination of processes. Frequent words in quadrant IV of Fig. 3 indicate this orientation: «affect», «effect», «reduce», «predator-induced», «impact», «interactive», «mediated», «trade-off», «alter» or «delayed». This trend agrees again with the findings of Swihart et al. (2002) and contradicts the criticism of O'Connor (2000) who argued that in ecology unlike biology, «how» questions are of marginal importance. 


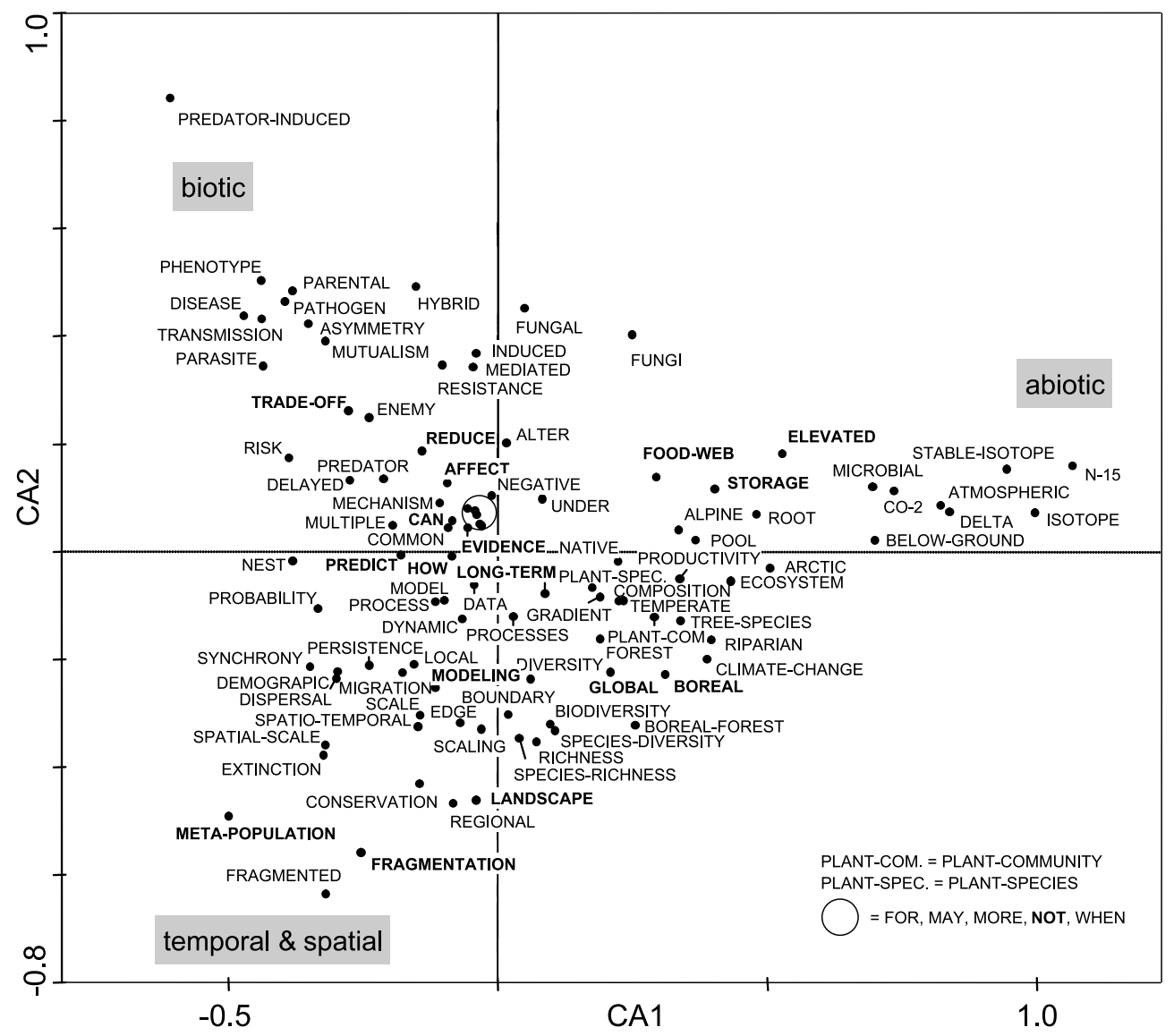

Fig. 5. CA-ordination of recent trend words (quadrant IV in Fig. 3) based on counts of co-occurrences in article abstracts. Transformation: $\mathrm{n}^{\prime}=\log (10 \times \mathrm{n}+1)$; the first and the second CA-axes accumulate $13.3 \%$ and $7.6 \%$ of total inertia (ti $\left.=0.374\right)$, respectively. Words in bold correspond to top words in Fig. 3.

The majority of frequent title words show a positive trend (Fig. 3). Despite the fact that we used relative counts, these outcomes can be explained, to some extent, by the general increase in number of articles and title words over the last 25 years (Fig. 1). In addition, the obvious concentration or globalization may result from the increased use of international literature that is fostered by literature databases, by an increasing international focus on few successful journals, and by an increased international orientation of high rated journals. Whereas successful (i.e. often cited) papers usually have an array of trend words in their titles, negative indicators of globalization may be geographical words, e.g. visible in Fig. 2 and 3: «Scotland», «Chilean», «Chile», «Sonoran», «Southern». In our data-sets, the dynamics of negative trends seem to be less correlated with time than the dynamics of positive trends; there are fewer title words that continuously fade out with time or vanish at all.

As shown in Fig. 3, title words are located in the neighbourhood of the publication years according to their highest relative frequencies and in accordance with the underlying linear model. Because of slightly fluctuating years in an imperfect horseshoe the assignment of these words to a single year may not be overrated. In addition, a slight increase of absolute counts of title words may be reversed by using relative frequencies. It is conspicuous, however, that expressions such as «ecosystem», «diversity» or «plant-species» (no. 148, 149 and 150) are located beyond the general time gradient (Fig. 3). The word «ecosystem» was more frequently used at the beginning and at the end of the gradient (Appendix 1), resulting in its intermediate position in the bi-plot. The same holds for the words «diversity» and «plant-species», though with a strong overbalance towards the end of the gradient. The expressions can be considered evergreens or bandwagons (Paine 2002): the words have been used for a long time in ecology and still became more important. About 19\% of all occurrences of «plant-species» refer to either «plant species diversity» or «plant species richness», as an effect of the booming «biodiversity» - a word that first appeared in a journal 
in 1987 (Wilson 1987) and that was derived from the expressions «biological diversity» and «bio-diversity» in the early 1980ies (Harper and Hawksworth 1994).

According to the results of the co-word analysis 1, trendy fields in ecology can be derived from words used in publication titles and abstracts. Despite the fact that only 66 words were used, the arrangement of at least 8 clusters allow for a plausible interpretation. In a first trend cluster (Fig. 4), words such as «scale» and «fragmentation» are related to «landscape». As the bars indicate, most of these words are new to the ecological core journals, implying that «landscape ecology» developed into one of the big new sub-disciplines in ecology, whether or not it has evolved from or invaded ecology. A second clear cluster concerns «biodiversity» and related words. The bars reveal that "diversity», «plant-species» and «species-richness» were already an issue in ecological journals 25 years ago. This cluster type agrees with the gradual model refinement (Graham and Dayton 2002) or with the conceptual evolution (Paine 2002) of a wide-ranging abstract concept (Gaston 1996). The position of «invasion» in close vicinity of biodiversity indicates both, a disciplinary relationship and the actual topicality of «species invasion» (Kolar and Lodge 2001). The third clear cluster consists of an array of words used in «ecosystem research». Old words in this category are «CO-2», «ecosystem» and «temperate» (often used as co-words of forest, Fig. 5). New are the words «climate-change» and «global». In this cluster type, the growing evidence of climate change (environmental anomaly) has joined a bandwagon subject of ecology (Cramer et al. 2001). Additionally, «stable isotope» and «N-15» indicate the influence of a methodological innovation. In the fourth cluster the highest frequency is found for «model» that is linked to «dynamic», «meta-population», "predict» or «spatiotemporal». Models have been used in ecology for a long time and are considered part of the scientific progress (Lomnicki 1988). However, the fast evolution of numerical methods and computing power have boosted the application of model approaches. The appearance of «meta-population» in this cluster shows that the metapopulation approach (Hanski and Gilpin 1991) gained greatly in importance during the 1990s still proves to be a topicality in ecology. The next obvious ecological cluster consists of two closely related bandwagons: «food-web» (Paine 2002) and predator-prey interactions indicated by «predator» (Johnson and Agrawal 2003). The importance of food webs may be best explained by its role in conservation biology (Pimm 2002). An additional interesting branch concerns the group of «affect», «how», «alter» and «can» that corresponds to the already mentioned trend towards the examination of processes. To a smaller degree, two branches at the bottom of the dendrogram exhibit strongly focused topicalities within ecology: «disease», «transmission», «parasite» and «atlantic», «salmon», «riparian». It is obvious to us, that the here applied classification approach merely connect the trend words found and that the sub-division of groups may differ according to the methods applied. A finer degree of sub-division could be approached by using larger samples. However, using a different approach in the co-word analysis 2, the cluster pattern reappears in Fig. 5 in the CA-ordination plot. In addition to the dendrogram, more words are included here and their relative positions are quite meaningful. As a methodical effect that can be often observed in CA, the scatterplot has a triangular structure. Surprisingly or not, the words towards the three corners reveal well-defined semantic fields of ecological research. The «biotic» sector at the top is based on expressions of biotic interactions (e.g. «parasite», «predator»), biotic characteristics (e.g. «phenotyp», «hybrid») and corresponding words (e.g. «transmission»). Most expressions fit in the broad field of community ecology and biocenology (Morin 1999, Kratochwil and Schwabe 2001) dealing with interacting organisms in ecosystems. Zoological expressions also relate to behaviour ecology. The «abiotic» sector at the right shows expressions such as «stable-isotope», «N-15», «delta», «elevated», « $\mathrm{CO}_{2}$ » and «atmosphere». The words represent trend aspects of ecophysiology and ecosystem ecology, dealing with partitioning and fluxes of abiotic resources in ecosystems. The «temporal and spatial» sector at the bottom is defined by expressions related to topics of landscape ecology, e.g. «fragmentation», «meta-population», «spatio-temporal» and «conservation».

Despite the often complained fragmentation of ecological research into sub-disciplines with a high degree of specialization, the arrangement of the semantic fields makes clear that sub-disciplines in ecology are still closely related. As an example, along the gradient from the «abiotic» to the «temporal and spatial» sector, we encounter «atmospheric», «CO-2», «climate-change», «species-richness», «conservation». Other arbitrarily chosen sections of the scatterplot would reveal similar meaningful connections among the different subdisciplines in ecology. Expressions found at the centre of the scatterplot are in general important and integrative for all three directions mentioned, e.g. «evidence», «how», «data», «model», «processes», «predict».

Astonishingly, the underlying structure of the semantic map corresponds quite well with the self-conception of ecology since the first definition by Haeckel (1866): ecology as a science dealing with systems characterized by biotic and abiotic attributes in space and time. In a first consideration, this arrangement could be interpreted as the often claimed lack of progress in ecology because the structure displayed here corresponds widely with the old definition. Looking carefully, however, all displayed words are trend words, and some of them have 
been introduced over the last 25 years characterizing new methods, advanced research fields and deeper insights (Fig. 3-5, Appendix 1). It is remarkable that, nevertheless, the trend words found cover the three main aspects of ecological research and that there is nothing like a dominating sub-discipline. We suggest that the situation reflected by the arrangement of trend words represents real scientific progress in ecology as a pluralistic, multi-aspect and multiple-causation discipline - scientific progress that could hardly be observed from details in sub-disciplines.

\section{Limitations and future prospects}

Interpretations of our results must be tempered owing to several factors discussed below:

\section{Selection of journals}

The selection of the five international and high rated ecological core journals results only in an approximate overview of the broad field of ecology. As a consequence biases are obvious. Appendix 1 shows distinct journal styles and disciplinary subjects: the question «how» is increasingly posed in the journals of «Ecology», "Oecologia» and «Oikos», whereas this trend is not conspicuous in the two British journals. Ecosystem functioning is a clear main subject of «Oecologia» and is best mirrored by the dramatic increase in the word «elevated» that most often co-occurs with both $\left\langle\mathrm{CO}_{2}\right.$ » and «atmospheric». More balanced analyses should take into account a higher number of international journals with ecology in the editorial outline.

\section{Brand-new trends}

We used the PCA for trend analyses, i.e. a method that implicates a linear distribution model. However, the use of title words indicating newer trends such as «metapopulation» or «landscape» (Appendix 1) partly began only during the observation period and increased exponentially in the beginning. A linear model can only approximately fit this kind of trend and results in moderate fits for these trend words. In addition, our approach was not sensitive enough to detect the most recent trends due to constraints of word frequencies. Despite their exponential increase, the time has usually been too short for brand-new trend words to reach top accumulation with respect to the 25-year period. Looking through the filtered eyes of the ISI Web of science, a considerable amount of publications in young fields remains hidden for a few years in brandnew journals. Because the citation index refers to references of the past two years, the brand-new journals only appear with delay in the ISI Web (Graham and Dayton 2002).
Acknowledgements - We are very grateful to Christian Körner, Michael W. Palmer, Robert K. Peet and Otto Wildi who contributed with valuable comments on earlier drafts of the manuscript, and to Sucharita Ghosh Beran who improved the English.

\section{References}

Aarssen, L. W. 1999. Progress drive from progress pride. - Oikos 85: 375-376.

Ambasht, R. S. 1998. Modern trends in ecology and environment. - Backhuys Publishers.

Budilova, E. V., Drogalina, J. A. and Teriokhin, A. T. 1997. Principal trends in modern ecology and its mathematical tools: an analysis of publications. - Scientometrics 39: $147-$ 157.

Cherrett, J. M. 1989. Key concepts: the results of survey of our member's opinions. - In: Cherrett, J. M. (ed.), Ecological concepts: the contribution of ecology to an understanding of the natural world. Blackwell Scientific, pp. 1-16.

Cramer, W., Bondeau, A., Woodward, F. I. et al. 2001. Global response of terrestrial ecosystem structure and function to $\mathrm{CO}_{2}$ and climate change: results from six dynamic global vegetation models. - Global Change Biol. 7: 357-373.

Gaston, K. J. 1996. What is biodiversity? - In: Gaston, K. J. (ed.), Biodiversity: a biology of numbers and differences. Blackwell Science, pp. 1-9.

Ghilarov, A. 1996. What does 'biodiversity' mean - scientific problem or convenient myth? - Trends Ecol. Evol. 11: 304306.

Ghilarov, A. M. 2001. The changing place of theory in 20th century ecology: from universal laws to array of methodologies. - Oikos 92: 357-362.

Graham, M. H. and Dayton, P. K. 2002. On the evolution of ecological ideas: paradigms and scientific progress. - Ecology 83: 1481-1489.

Grime, J. P. 1993. Ecology sans frontieres. - Oikos 68: 385-392. Haeckel, E. 1866. Generelle Morphologie der Organismen. - Reimer.

Hanski, I. and Gilpin, M. 1991. Metapopulation dynamics: brief history and conceptual domain. - Biol. J. Linn. Soc. 42: 3-16.

Harper, J. L. and Hawksworth, D. L. 1994. Biodiversity: measurement and estimation. - Philos. Trans. R. Soc. Lond. Ser. B Biol. Sci. 345: 5-12.

Johnson, M. T. J. and Agrawal, A. A. 2003. The ecological play of predator-prey dynamics in an evolutionary theatre. - Trends Ecol. Evol. 18: 549-551.

Keeling, C. D., Bacastow, R. B., Bainbridge, A. E. et al. 1976. Atmospheric carbon-dioxide variations at Mauna-Loa observatory, Hawaii. - Tellus 28: 538-551.

Kolar, C. S. and Lodge, D. M. 2001. Progress in invasion biology: predicting invaders. - Trends Ecol. Evol. 16: 199204.

Körner, C. and Spehn, E. M. 2002. Mountain biodiversity: a global assessment. - Parthenon.

Kratochwil, A. and Schwabe, A. 2001. Ökologie der Lebensgemeinschaften: Biozönologie. - Ulmer.

Kuhn, T. S. 1962. The structure of scientific revolutions. - Univ. of Chicago Press.

Lawton, J. H. 1991. Ecology as she is done, and could be done. - Oikos 61: 289-290.

Lawton, J. H. 1999. Are there general laws in ecology? - Oikos 84: $177-192$.

Lomnicki, A. 1988. The place of modeling in ecology. - Oikos 52: $139-142$.

Manel, S., Schwartz, M. K., Luikart, G. et al. 2003. Landscape genetics: combining landscape ecology and population genetics. - Trends Ecol. Evol. 18: 189-197. 
Maruyama, N., Bobek, B., Ono, Y. et al. 1991. Wildlife conservation: present trends and perspectives for the 21th century. - Wildlife Research Center.

Morin, P. J. 1999. Community ecology. - Blackwell Sciences.

Naeem, S. 2002. Ecosystem consequences of biodiversity loss: the evolution of a paradigm. - Ecology 83: $1537-1552$.

Nagy, L., Grabherr, G., Körner, C. et al. 2003. Alpine biodiversity in Europe: analysis and synthesis. - Springer.

O'Connor, R. J. 2000. Why ecology lags behind biology. - Scientist 14: 35-35.

Odén, S. 1968. The acidification of air and precipitation and its consequences in the natural environemnt. - Ecol. Committee Bull. Swedish Natl Sci. Res. Council.

Oksanen, L. 1991. A century of community ecology - how much progress? - Trends Ecol. Evol. 6: 294-296.

Oksanen, T., Oksanen, L. and Persson, L. 2001. Costs and gains of recent progress in ecology - Proc. Oikos seminar held at Hallnas, Sweden, October 1999 - Introduction to the symposium. - Oikos 94: 4-5.

Paine, R. T. 2002. Advances in ecological understanding: by Kuhnian revolution or conceptual evolution? - Ecology 83: $1553-1559$.

Peters, R. H. 1991. A critique for ecology. - Cambridge Univ. Press.
Pimm, S. L. 2002. Food webs. - Univ. Press of Chicago.

Podani, J. 1994. Multivariate data analysis in ecology and systematics. A methodological guide to the SYN-TAX 5.0 package. - SPB Academic Publishing.

Shorrocks, B. 1993. Trends in the Journal-of-Animal-Ecology 1932-92. - J. Anim. Ecol. 62: 599-605.

Shurin, J., Gergel, S., Kaufman, D. et al. 2001. In defense of ecology. - Scientist 15: 6-6.

Swihart, R. K., Dunning, J. B. and Waser, P. M. 2002. Gray matters in ecology: dynamics of pattern, process, and scientific progress. - Bull. Ecol. Soc. Am. 83: 149-155.

ter Braak, C. J. F. and Smilauer, P. 2002. CANOCO reference manual and CanoDraw for Windows user's guide: software for canonical community ordination (version 4.5). - Biometris.

Weiner, J. 1995. On the practice of ecology. - J. Ecol. 83: 153158.

Weiner, J. 1999. On self-criticism in ecology. - Oikos 85: $373-$ 374.

Wiens, J. A. 1992. Ecology 2000: an essay on future directions in ecology. - Bull. Ecol. Soc. Am. 73: 165-170.

Wilson, E. O. 1987. An urgent need to map biodiversity. - Scientist 1: 11-11.

Woodward, F. I. 1987. Plant ecology - trends and traits. - Trends Ecol. Evol. 2: 252-254. 


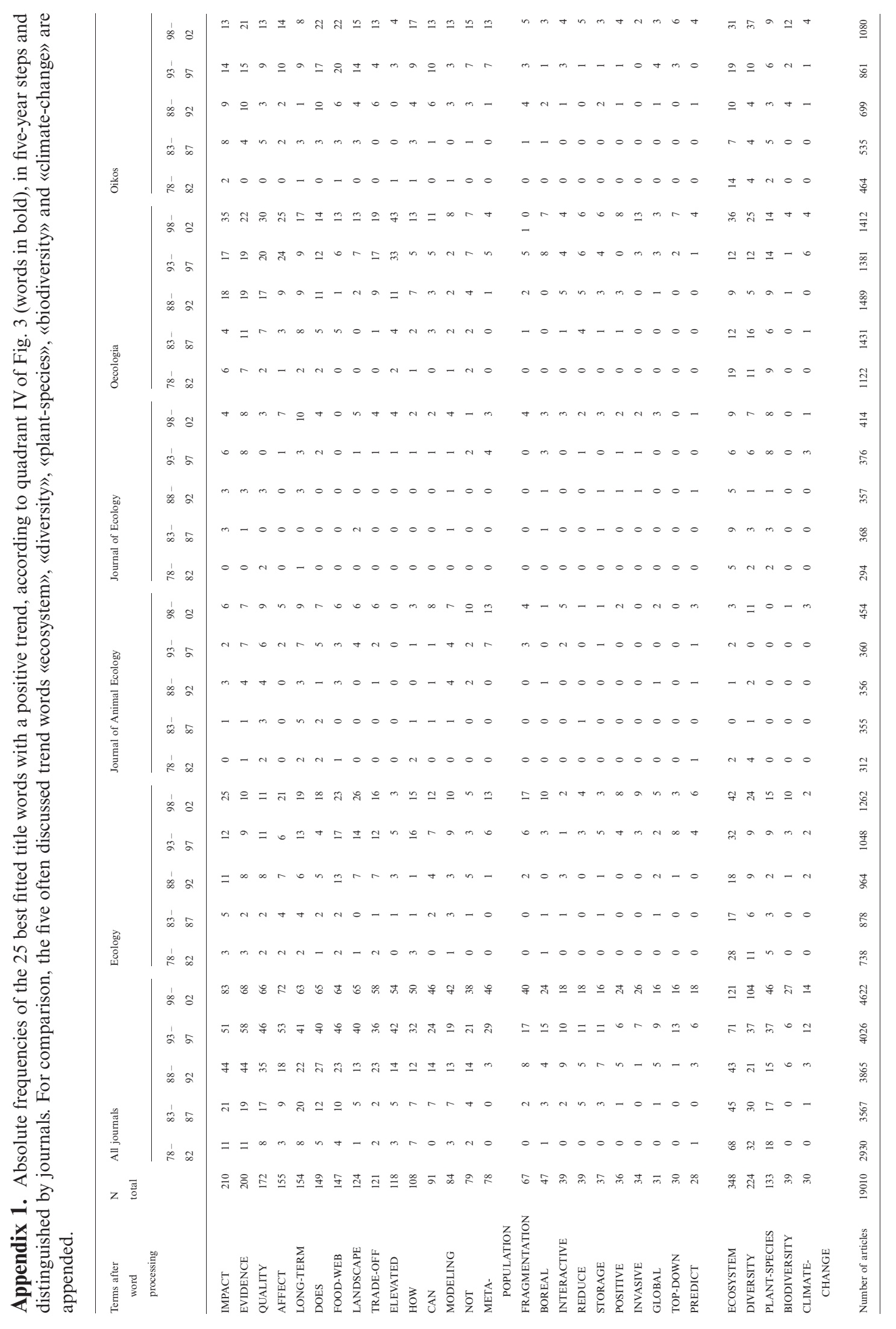

OIKOS 106:2 (2004) 